\title{
Experimental Study on Flow Characteristics of Open-channel Flow with Flexible Vegetation
}

\author{
Yang $\mathrm{CHEN}^{1,2, \mathrm{a}}$, Jing $\mathrm{YAN}^{1,2, \mathrm{~b},{ }^{*},}$, Ye HE ${ }^{3, \mathrm{c}}$, Kun DAI ${ }^{1,2, \mathrm{~d}}$, Ming ZHANG ${ }^{1,2}$ \\ ${ }^{1}$ State Key Laboratory of Hydrology-Water Resources and Hydraulic Engineering, Nanjing 210098, \\ China \\ ${ }^{2}$ College of Water Conservancy and Hydropower, Hohai University, Nanjing 210098, China \\ ${ }^{3}$ Bureau of Water Conservancy of Jian Hu County, Jian Hu County, 224700, China \\ a653583040@qq.com, byanjing@hhu.edu.cn, c493111645@qq.com, ${ }^{\mathrm{d}} 786437718 @ q q . c o m$ \\ ${ }^{*}$ Corresponding author
}

\begin{abstract}
Keywords: Flexible Vegetation, Flow Characteristics, Mean Bulk Velocity, Submergence, Vegetation Density.
\end{abstract}

\begin{abstract}
Laboratory experiments with silicone cylinders simulating flexible vegetation, the Acoustic Doppler velocimetry (ADV) measuring the flow field were performed to investigate the turbulence characteristics of open-channel flow with flexible vegetation. The results and analysis indicate that mean bulk velocity, submergence and vegetation density have significantly effect on streamwise velocity, turbulence intensity and Reynolds stress of flow respectively.
\end{abstract}

\section{Introduction}

As an important part of the river ecosystem, aquatic vegetation has an irreplaceable role. The basic flow characteristics mainly include velocity, turbulence intensity and Reynolds stress. Studies have shown that turbulence characteristics of open-channel flow with vegetation vary greatly with those without vegetation [1]. Temple [2] divided the vertical profile of streamwise velocity into two regions based on the mixing length theory. Wilson [3] simulated two forms of plants, which were flexible rods and same rods with a frond foliage attached according to Froudian Similarity and carried out comparison experiments to study the effect of leaves on Reynolds stress. For flow turbulence characteristics, main concerns of most studies are the description for velocity profile $[4,5,6]$, the position of the maximum value and profile form of turbulence intensity and Reynolds stress[7,8,9]. This paper selected silicone cylinders to simulate flexible vegetations and applied Acoustic Doppler velocimeter (ADV) to study the effects of mean bulk velocity $U m$, submergence depth $H / H v$ and vegetation density $I$ on the profile of velocity, turbulence intensity and Reynolds stress.

\section{Experimental Setup}

Three series of experiments, which were used to examine the effects of mean bulk velocity $U m$, relative submergence depth $H / H v$ and vegetation density $I$ on the turbulence characteristics respectively, were conducted in a $12 \mathrm{~m}$ long and $42 \mathrm{~cm}$ wide variable slope rectangular glass flume. Silicone cylinders were used as elements of simulated vegetation. The size of one vegetation element was $h=9 \mathrm{~cm}$ height, $d_{v}=6 \mathrm{~mm}$ diameter. The elements were fixed onto the gray plastic plate, which was installed on the bottom of flume. The vegetation density $I$ was defined as the total number of elements per square meter.

Flow was measured with ultrasonic flowmeter installed in the inlet piping. The element, which was $7 \mathrm{~m}$ downstream of inlet in the centerline, was selected as reference element. Instantaneous velocities of two vertical lines were measured by acoustic Doppler velocimetry (ADV), and the sampling frequency and time of which were $200 \mathrm{~Hz}$ and 30s per measuring point, respectively. One line was located in point $\mathrm{A}$, which was $1 \mathrm{~cm}$ away from the reference element along the flow direction. The other was located in point $\mathrm{B}$, which was also $1 \mathrm{~cm}$ away from it along the spanwise direction. The two sets of data were averaged to describe the flow field characteristics. 
Table 1 shows the hydraulic conditions, in which $F r=U m / \sqrt{g H}$ is Froude number and $\operatorname{Re}=U m H / v$ is the Reynolds number. $x, y$ and $z$ are streamwise, vertical and spanwise coordinates, respectively. $U$ denotes streamwise mean velocity, $u, v$ denotes velocity fluctuations of $x$ and $y$ coordinates, $u$ ' denotes the r.m.s value, i.e., turbulence intensity.

Tab. 1 Hydraulic Conditions for Present Experiments

\begin{tabular}{cccccccccc}
\hline Case & $H(\mathrm{~cm})$ & $Q(1 / \mathrm{s})$ & $S(1 / 10000)$ & $U_{m}(\mathrm{~cm} / \mathrm{s})$ & $\begin{array}{c}u * S R \\
(\mathrm{~cm} / \mathrm{s})\end{array}$ & $\operatorname{Re}\left(10^{4}\right)$ & $F r$ & $\begin{array}{c}I \\
\left(\text { elements } / \mathrm{m}^{2}\right)\end{array}$ & $H / H v$ \\
\hline $\mathrm{A} 1$ & 27 & 28.35 & 4.6 & 25 & 2.32 & 2.92 & 0.152 & 476 & 3.0 \\
$\mathrm{~A} 2$ & 27 & 22.7 & 3.9 & 20 & 2.13 & 2.34 & 0.122 & 476 & 3.0 \\
$\mathrm{~A} 3$ & 27 & 17.01 & 3.7 & 15 & 2.08 & 1.75 & 0.091 & 476 & 3.0 \\
$\mathrm{~A} 4$ & 22.5 & 18.9 & 4.7 & 20 & 2.25 & 2.17 & 0.133 & 476 & 2.5 \\
$\mathrm{~A} 5$ & 18 & 15.12 & 6.3 & 20 & 2.46 & 1.94 & 0.149 & 476 & 2.0 \\
$\mathrm{~B} 1$ & 27 & 28.35 & 5.8 & 25 & 2.60 & 2.92 & 0.152 & 381 & 3.0 \\
$\mathrm{C} 1$ & 27 & 28.35 & 7.0 & 25 & 2.85 & 2.92 & 0.152 & 190 & 3.0 \\
\hline
\end{tabular}

\section{Results and Discussion}

\section{Effects of Mean Bulk Velocity on Turbulence Characteristics}

Effects on Streamwise Mean Velocity. Fig. 1shows the vertical profiles of streamwise mean velocity $U(y)$ of Cases A1, A2, A3. The values of $U$ are normalized by friction velocity $u *_{S R}[6]$ because of the presence of vegetation. Besides, the values of $y$ are normalized by vegetation height $H v$. A inflection point of vertical profile appears on the top of canopy $(y / H v=1)$. As the mean bulk velocity becomes large, the vertical profiles of streamwise mean velocity becomes flat. In other word, the difference of velocity between the upper of canopy and the canopy becomes large. Since the drag effect of canopy, the mean bulk velocity has a little effect on the velocity in the canopy $(y / H v<1)$, on the contrary, the velocity above the canopy is larger with larger mean bulk velocity.

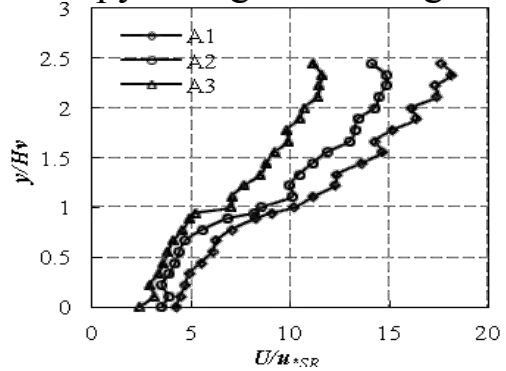

Fig. 1 The Vertical Profiles of Streamwise Mean Velocity

Effects on Turbulence Intensity and Reynolds Stress. (1) Effects on Turbulence Intensity. Fig. 2(1) shows the vertical profiles of turbulence intensity u'(y) of Cases A1, A2, A3. Turbulence intensity reaches maximum near the top of canopy, and decreases gradually toward the bed and water surface respectively. Turbulence intensity increases with increasing mean bulk velocity. This is because flexible vegetation deflected more significantly by the increasing mean bulk velocity so that the degree of flow disturbance is enhanced.

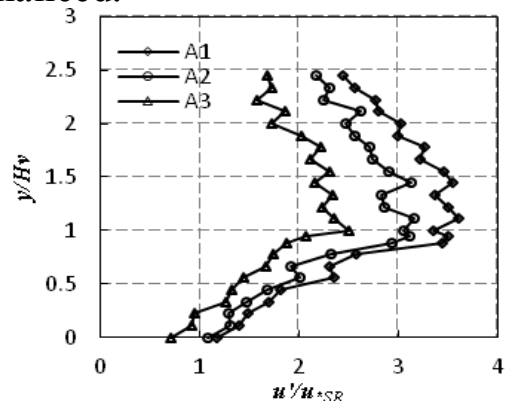

Fig. 2(1) The Vertical Profiles of Turbulence Intensity 
(2) Effects on Reynolds Stress. The profiles of Reynolds stress, $-u v(y)$, of three Cases A1, A2, A3 was shown in Fig. 2(2). The values of $-u v$ are normalized by the square of friction velocity, $u^{2}{ }_{* S R}$. The profiles of Reynolds stress are consistent with those of turbulence intensity. Reynolds stress also reaches maximum near the top of canopy, and decreases gradually toward the bed and water surface respectively. The effects of mean bulk velocity on Reynolds stress are apparent. As the mean bulk velocity becomes large, the values of Reynolds stress become large, which is significant above the canopy.

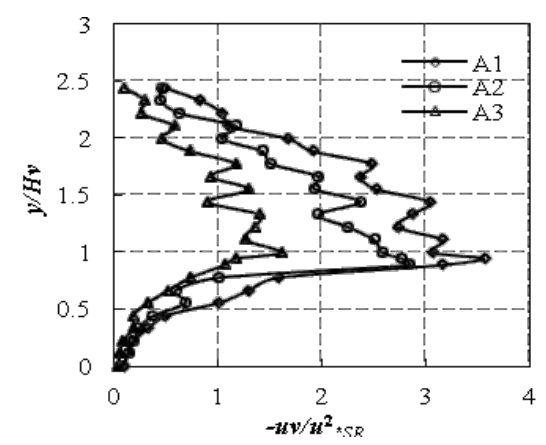

Fig. 2(2) The Vertical Profiles of Reynolds Stress

\section{Effects of Submergence Depth on Turbulence Characteristics}

Effects on Streamwise Mean Velocity. The vertical profiles of velocity of different submergence depths, Cases A2, A4, A5, are shown in Fig. 3. The overall velocity increases with the decrease of submergence depth, $H / H v$, and the vertical profile of velocity is steeper for Case A5, especially in the canopy.

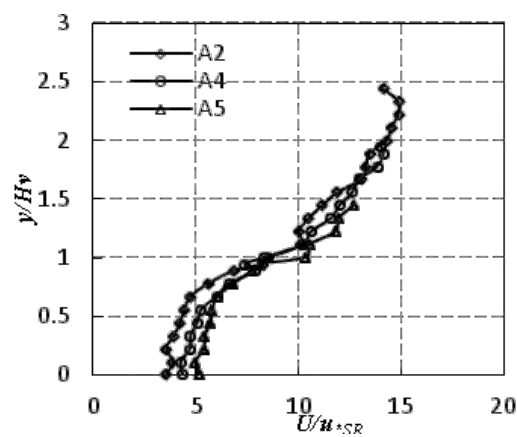

Fig. 3 The Vertical Profiles of Streamwise Mean Velocity

Effects on Turbulence Intensity and Reynolds Stress. (1) Effects on Turbulence Intensity. Fig. 4(1) shows the vertical profiles of turbulence intensity $u$ ' $(y)$ of Cases A2, A4, A5. The turbulence intensity above the canopy is larger in larger submergence depth, however, those in the canopy have a little change. The reason may be that the increase of submergence depth leads the increase of dominant degree of $\mathrm{K}-\mathrm{H}$ vortex.

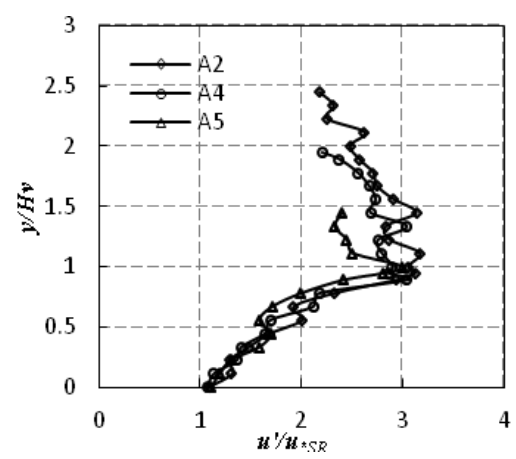

Fig. 4(1) The Vertical Profiles of Turbulence Intensity 
(2) Effects on Reynolds Stress. Fig. 4(2) shows the vertical profiles of Reynolds Stress $-u v(y)$ of Cases A2, A4, A5. With the increase of submergence depth $H / H v$, a significant increase of Reynolds stress appears above the canopy.

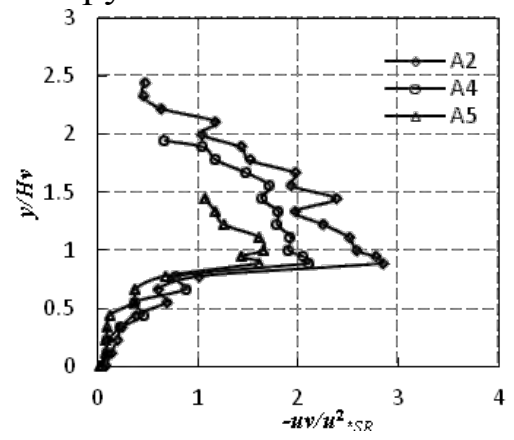

Fig. 4(2) The Vertical Profiles of Reynolds Stress

\section{Effects of Vegetation Density on Turbulence Characteristics}

Effects on Streamwise Mean Velocity. The vertical profiles of velocity of different vegetation density, Cases A1, B1, C1, are shown in Fig. 5. As one of important vegetation factor, vegetation density has direct effects on the profiles of velocity. The velocity above the canopy increases with the increase of vegetation density because the drag effects of vegetation decelerate the velocity in canopy. In the vegetation layer, the velocities of three densities are more concentrated.

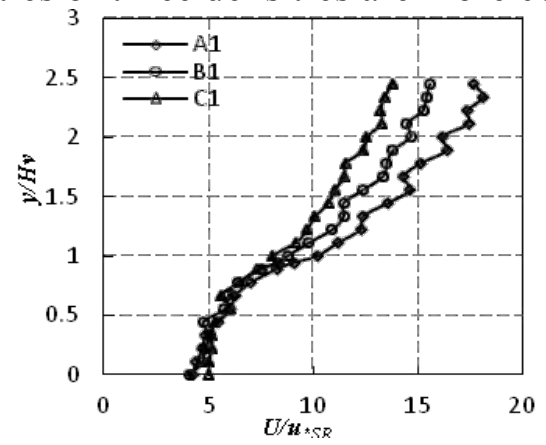

Fig. 5 The Vertical Profiles of Streamwise Mean Velocity

Effects on Turbulence Intensity and Reynolds Stress. (1) Effects on Turbulence Intensity. Fig. 6(1) shows the vertical profiles of turbulence intensity $u$ ' $(y)$ of Cases A2, A4, A5. The turbulence intensity above the canopy is especially larger in the denser vegetation. This infers that the denser vegetation density has significant effect on the increase of turbulence intensity.

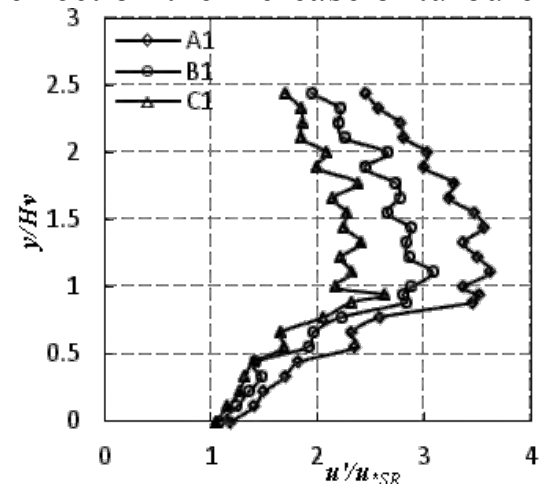

Fig. 6(1) The Vertical Profiles of Turbulence Intensity

(2) Effects on Reynolds Stress. Fig. 6(2) shows the vertical profiles of Reynolds stress $-u v(y)$ of Cases A2, A4, A5. As vegetation density increases, the Reynolds stress increases, which is consistent with the variation of turbulence intensity. 


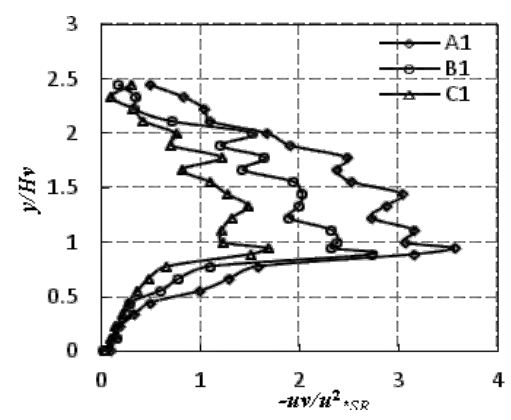

Fig. 6(2) The Vertical Profiles of Reynolds Stress

\section{Summary}

This paper applied Acoustic Doppler velocimetry (ADV) to study the turbulence characteristics of open-channel flow with flexible vegetation. The significant results obtained in this study are as follows: The inflection point of vertical profile of velocity appears on the top of canopy. With the increase of mean bulk velocity, submergence depth, vegetation density, the profiles of velocity become flat, this result is significant above the canopy. The profiles of turbulence intensity and Reynolds stress are similar. They both obtain the maximum value near the top of canopy, and increase with the increase of mean bulk velocity, submergence depth, vegetation density.

\section{Acknowledgement}

This research was financially supported by the National Natural Science Foundation of China (Grant No: 51109066, 51309083, 51125034, 51479070) and the National Basic Research Program of China (973 Program) (NO.2011CB403303).

\section{References}

[1]Lv Shengqi, Tang Hongwu, Yan Jing. Comparison of turbulence characteristics of water flow in open channels with and without vegetations.[J] Advances in Science and Technology of Water Resources, 2007,(06):64-68.

[2]Temple D M. Velocity distribution coefficients for grass-lined channels [J]. J.Hydreucl.Eng., 1986, 112(3): 193-205.

[3]Wilson C.A.M.E, Stoesser T, Bates P.D, et al. Open channel flow through different forms of submerged flexible vegetation [J].Journal of Hydraulic Engineering, 2003, 129(11):847-853.

[4]Carollo F G, Ferro V, Termini D. Flow Velocity measurements in vegetated channels [J]. Journal of Hydraulic Engineering, 2002, 128(7):664-673.

[5]Nezu, I, and Sanjou. M. 2008. Turbulence structure and coherent motion in vegetated canopy open-channel flows, J. of Hydro-environment Research, 2, 62-90.

[6]Yan Jing, Tang Hongwu, et al. Experimental study on the influence of vegetation on the velocity distribution of open channel flows [J]. Hydro-Science and Engineering, 2011, (04):138-142.

[7]Li Yanhong, Li Dong, Fan Jinglei. Turbulence intensity maximum and its influence factors in submerged river flow with plant [J].Advance in Water Science. 2007, (05):706 -710.

[8]Huai Wenxin, Han Jie, et al. Experimental study on hydraulic behaviors of steady uniform flow in open channel with submerged flexible vegetation [J]. Journal of Hydraulic Engineering. 2009.(07): 791-797.

[9]Yan Jing. Experimental study on flow resistance and turbulence characteristics of open channel flows with vegetation [D]. Dissertation of Doctor degree of Hohai university, 2008, Nanjing. 